The Journal of Animal \& Plant Sciences, 31(2): 2021, Page: 488-499
ISSN (print): 1018-7081; ISSN (online): 2309-8694

\title{
TISSUE CULTURE OPTIMIZATION OF PODOPHYLLUM HEXANDRUM L., AN ENDANGERED MEDICINAL PLANT
}

\author{
Z. Zuhra ${ }^{1}$, D. Saleem ${ }^{1}$, W. Akhtar ${ }^{2}$ and T. Mahmood ${ }^{1 *}$ \\ ${ }^{1}$ Department of Plant Sciences, Quaid-i-Azam University, Islamabad, Pakistan \\ ${ }^{2}$ Department of Botany, University of Azad Jammu \& Kashmir, Muzaffarabad, Pakistan \\ *Corresponding author: tmahmood.qau@gmail.com;tmahmood@qau.edu.pk
}

\begin{abstract}
P. hexandrum (Bankhakri) is an important medicinal plant due to its useful active compounds such as podophyllotoxin, a precursor of anti-cancer drugs. Currently, it is carrying the status of endangered species in Pakistan, India, China and Afghanistan due to slow growth and overharvesting. The present study is an attempt to establish an efficient protocol for callogenesis and organogenesis of $P$. hexandrum. Fresh fruits and rhizomes were collected from natural habitats. Seeds were sterilized and germinated for callogenesis and organogenesis. The present study describes that leaf explants resulted in the highest callus response $(100 \%)$ followed by stem $(90 \%)$ on Murashige and Skoog media (MS) media supplemented with $6.75 \mu \mathrm{M}$ Naphthalene acetic acid (NAA) and 11.37 $\mu \mathrm{M}$ 6-Benzylaminopurine (BAP). Moreover, 1.0 $\mu \mathrm{M}$ indole acetic acid (IAA) in combination with $5.0 \mu \mathrm{M}$ BAP successfully induced $80 \%$ callogenesis from root explants while 2,4 Dichloro-phenoxy acetic acid (2,4-D) at 3.0 and $4.0 \mu \mathrm{M}$ resulted in $20 \%$ calli from embryos. Principal component analysis (PCA) showed that leaf and stem explants revealed considerable callus response in combination of NAA+BAP. Rhizome did not support callogenesis rather direct shooting was achieved with a combination of IAA $(10.42-11.42 \mu \mathrm{M})$ and activated charcoal $5 \mathrm{~g} / \mathrm{L}$. ANOVA showed the most significant variability $(P=0.007198)$ from rhizome explant in direct regeneration.
\end{abstract}

Keywords; Podophyllum hexandrum L., Tissue culture, Conservation, Callogenesis, Organogenesis. https://doi.org/10.36899/JAPS.2021.2.0238

Published online October 03,2020

\section{INTRODUCTION}

P. hexandrum L. Syn. P. emodi Wall. of family Berberidaceae is commonly called Bankhakri in Pakistan due to its unique fruit (Phondani et al., 2010). This rhizomatous perennial herb produces a solitary flower which blooms in May and sets berry like single fruit in September-October (Sreenivasulu et al., 2009; Selvam, 2012). It grows well in moist shady alpine meadows at $2000-4500 \mathrm{~m}$ altitudes in South Asia, Afghanistan, America and China (SHARMA et al., 2013). It can thrive a range of very low temperature of winter to extreme summer temperature (Selvam, 2012). In Pakistan, $P$. hexandrum is distributed at higher altitudes of Himalaya, Gilgit, Chitral and Azad Jammu and Kashmir (Chaudhari et al., 2014).

Its high-ranking medicinal significance is attributed to active compounds such as podophyllotoxin or podophyllin (neurotoxin), its glycosides, epipodophyllotoxin, podophyllotoxone, podophyllic acid and anti-mutagenic. Podophyllotoxin is a precursor of etoposide (Vepeside), approved anticancer drug by food and drug administration (FDA) and semi-synthetic topoisomerase inhibitors which are used to treat testicular and lung cancer (Giri and Narasu, 2000; Qazi et al., 2011; Dar et al., 2017). Podophyllotoxin derived pesticides are used to control crop attacking insect pests
(Yang et al., 2017). Interestingly, high-altitude cultivation and chilling triggers more podophyllotoxin accumulation (Sun and Li, 2016; Li et al., 2018).

Currently, $P$. hexandrum is endangered due to over harvesting of young plant parts to meet the market demands. Naturally, slow growth, lengthy juvenile phase, single fruit per plant per year and deficient fruit setting also add to its threatened status (Nadeem et al., 2000; Pandey et al., 2007). National Medicinal Plant Board of India (Chauhana et al., 2013) and International Union for Conservation of Nature and Natural Resources (IUCN) has banned its export (Kumari et al., 2017). Tissue culturing was reported to conserve $P$. haxandrum and invitro generated callus were used in anti-cancer assays (Heyenga et al., 1990; Chakraborty et al., 2010; Rajesh et al., 2014).

Despite of threatened rank, its export and harvesting have not been banned in Pakistan. Moreover, only RAPD based diversity was accessed from in-vitro cultured P. haxandrum (Tariq et al., 2015) and no comprehensive trials were done for tissue cultured based conservation in Pakistan. Over harvesting is a serious threat, so measures should be made to propagate and conserve it (Kharkwal et al., 2008; Qazi et al., 2011; Pandey et al., 2019). The present study was designed to establish a protocol for callogenesis and organogenesis of P. hexandrum. 


\section{MATERIALS AND METHODS}

Plant Collection: The fresh fruits and rhizome of $P$. hexandrum were collected from the non-timber forest of Ayubia, KP, Pakistan during May-September 2017 and 2018.The plant was identified with voucher number AKASH006061 in department of Botany University of Azad Jammu and Kashmir. The experiments were carried out in plant biochemistry and molecular biology lab at Quaid-i-Azam University Islamabad. In total ten numbers of fruits were collected from which about 350 seeds were separated and washed under tap water, shade dried overnight and stored at $4{ }^{\circ} \mathrm{C}$.

Seeds and Explants Sterilization: Seeds were first disinfected by an aqueous solution of $50 \%$ Sodium hypochlorite $(\mathrm{NaOCl})$ for 5 minutes and washed with autoclaved distilled water. Then seeds were soaked in 70 $\%$ ethanol for 2 minutes and washed with autoclaved distilled water. Similarly, rhizomes were washed with tap water and then immersed in $20 \%$ Tween-20 solution for 20 minutes to remove contaminants. Rhizome was sterilized with $0.2 \%$ aqueous solution of Mercuric chloride $\left(\mathrm{HgCl}_{2}\right)$ for 2-3 minutes. Then rhizome was treated with $70 \%$ ethanol for 8 minutes and washed with autoclaved distilled water.

Seed Dormancy Breakage and Germination: As $P$. hexandrum seeds remain dormant for whole season until favorable conditions arrive. So, seeds were given a strong pre-treatment like scarification to break dormancy. The stratified seeds were imbibed for 24 hours. About 100 seeds were sowed in peat moss soil containing equal ratio (1:1:1:1) of vermiculite, farmyard manure, sand and soil and placed under shady and humid conditions at 20-22 ${ }^{\circ} \mathrm{C}$. Seedling percentage was observed and recorded after regular intervals of 10 days. Leaves were emerged after 3 months.

In vitro Culturing: The plant shoots collected from ex situ conditions were sterilized and inoculated on MS media to gain more explants for callus culture. Leaves, stems, embryos and roots were used as explants. The explants were first washed with autoclaved distilled water and then cultured on MS media supplemented with different combinations and concentrations of PGR's under aseptic conditions.

Callogenesis: The embryos were precisely excised from seeds and explants were then inoculated on different culture media for callus induction. MS was fortified with fifteen different concentrations and combinations of PGR's (2,4-D, BAP and NAA) individually (Table 1). The culture conditions were optimized in a plant growth chamber (Thermostable GC-450) set at $25{ }^{\circ} \mathrm{C}$ and $60 \%$ humidity with photoperiod of $16 / 8 \mathrm{~h}$ light/dark respectively. Ten different concentrations and combinations of PGR's (NAA+BAP) were supplemented to MS for calli from leaf and stem explants (Table 2). Similarly, root tips and rhizome cuttings were also cultured on MS with ten different concentrations of auxins (IAA, NAA) individually and in combinations with cytokinins (NAA+BAP, IAA+BAP) for callus induction (Table 3). To increase the rate of callus induction, explants were sub-cultured on fresh media after regular intervals of two weeks and fully-grown calli were established after eight weeks.

\section{Organogenesis}

In-Direct Regeneration: The mature calli developed from embryo, leaf, stem, roots and rhizome explants were shifted to MS fortified with different combinations and concentrations of auxins and cytokinin (IAA 11.42 $\mu \mathrm{M}+\mathrm{BAP} 5.37 \mu \mathrm{M})$ for shoot initiation.

Direct Regeneration: Rhizome explants were also cultured on MS supplemented with activated charcoal 5 $\mathrm{g} / \mathrm{L}$ and IAA $(8.42-12.42 \mu \mathrm{M})$ in different concentrations for direct regeneration (Table 4). In-vitro regenerated shoots were then transferred to another media for root initiation. The $4-5 \mathrm{~cm}$ long shoots were then cultured on $1 / 2$ strength MS added with $1 \mathrm{~g} / \mathrm{L}$ activated charcoal and different concentrations of auxins NAA and IAA alone and their combinations. Cytokinin (BAP) was also used along with auxins in root induction (Table 5).

Acclimatization: The rooted plants were taken out from cultured pots and shifted to the soil pots containing sterile sand, soil and vermiculite mixture $(1: 1: 1)$. The plantlets were first kept under optimized greenhouse conditions for acclimatization and then placed in open environment under natural conditions and their survival rate were recorded on weekly basis.

Statistical Analysis and Experimental Design: Calli number, shooting and rooting percentage was noted on each media combination. Analysis of variance (ANOVA) was performed for callus induction, plant parts and hormonal combinations by Paleontological Statistics Software (PAST). PCA was conducted for callus induction from leaves, stem and roots on different media combinations. $P$ (same) value $<0.05$ was considered as observable in ANOVA. P value was also calculated using PAST normality test and values $<0.05$ considered as statistically significant and $<0.01$ as highly significant. The experiments were carried out in triplicates to get significance of study.

\section{RESULTS}

Seeds showed germination response only in $e x$ situ conditions after 4-8 weeks of sowing and no response was observed in in vitro culturing. Pre-treated seeds with $\mathrm{GA}_{3}(1000 \mathrm{ppm})$ and conc. $\mathrm{H}_{2} \mathrm{SO}_{4}$ showed $90 \%$ and 75 $\%$ response after stratification respectively. While $60 \%$ 
seeds germinated in natural conditions without pretreatments like control plants. Pre-treatments resulted in $30 \%$ increased germination (Table 6) and lead to $90 \%$ germination.

Callogenesis: The best callus was supported by leaf (100 $\%$ ) and stem explants (90\%) on $\mathrm{C}_{7 \mathrm{LS}}$ (NAA $6.75 \mu \mathrm{M}$ and BAP $11.37 \mu \mathrm{M}$ ) media respectively (Table 7). The maximum callogenesis $(80 \%)$ from root explants was observed with induction on media $\mathrm{C}_{9 \mathrm{R}}$ (IAA $1.0 \mu \mathrm{M}+$ BAP 5.0 $\mu \mathrm{M}$ ). However, rhizome did not induce the callogenesis (Table 8). The excised embryos promoted callogenesis only with $3-4 \mu \mathrm{M} 2,4-\mathrm{D}$ showing $20 \%$ efficiency after 8 weeks (Table 9). The size, color and texture of calli induced from different explants varied with concentrations and combinations PGRs (Figure 1). The efficient and frequent callus induction occurred from leaf explants with a mean percentage response of $76 \%$ as compared with stem $(71 \%)$, roots $(66 \%)$ and embryos (20\%). Overall, leaf explants showed the highest callus induction and embryo explants showed the lowest callus induction (Figure 2).

Statistical Analysis: Statistical analysis of callus responses of leaf, stem, roots and embryo explants with NAA, IAA, 2, 4-D, BAP and their combinations $(2,4-$ $\mathrm{D}+\mathrm{BAP}, \mathrm{IAA}+\mathrm{BAP}$ and $\mathrm{NAA}+\mathrm{BAP}$ ) was conducted by principal component analysis (PCA) using PAST. PCA analysis showed that leaf and stem explants revealed a significance response in a similar pattern. It was observed that hormonal combinations (NAA+BAP) are the most suitable for callus induction (Figure 3 ).

Organogenesis: All calli could not survive and regenerate even after 45 days. The shooting was observed from rhizome on all the shooting media of which $\mathrm{S}_{4}$ (IAA $11.42 \mu \mathrm{M}$ ) and $\mathrm{S}_{5}$ (IAA $12.42 \mu \mathrm{M}$ ) showed the maximum number (Table 10) of shoots as shown in Figure 4. Statistical analysis of one-way ANOVA was found to be highly significant $(P=0.007198)$ for direct regeneration from rhizome explants (Supplementary Table 1). Shoots were separately inoculated on different rooting media supplemented with (NAA, IAA, BAP) and activated charcoal. The efficient root induction was supported on media $\mathrm{R}_{5}$ (IAA $5.0 \mu \mathrm{M}+\mathrm{BAP} 0.5 \mu \mathrm{M}$ ) and $\mathrm{R}_{6}$ (IAA 5.5 $\mu \mathrm{M}+\mathrm{BAP} 0.5 \mu \mathrm{M}$ ) (Table 11). The plantlets with developed roots were transferred to the soil pots containing equal ratio $(1: 1: 1: 1)$ of vermiculite, farmyard manure, sand and soil. The plants were kept under greenhouse conditions and then established plants were shifted to natural conditions and their survival rate was recorded on weekly basis.

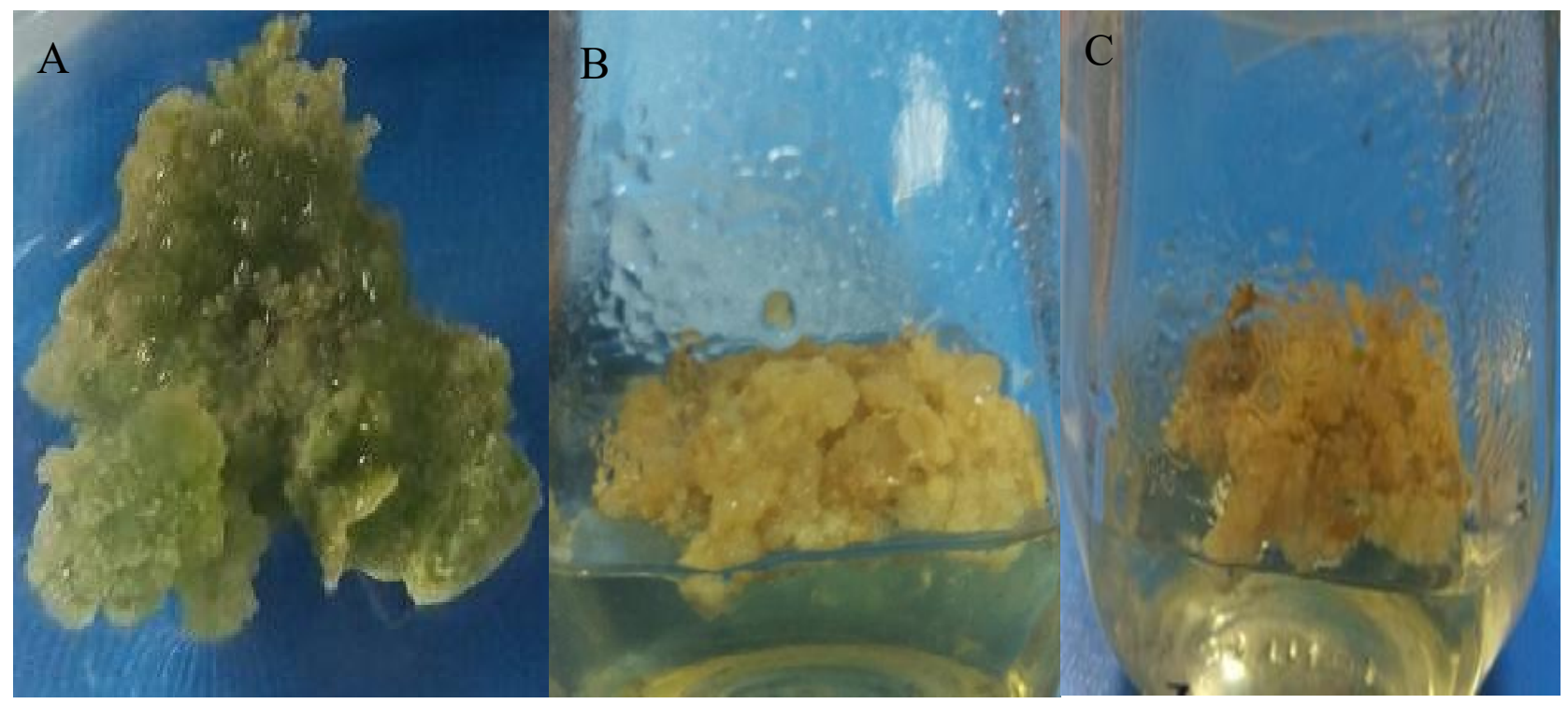

Figure 1. Calli induced from different explants. (A) Leaf callus, (B) Stem callus, (C) Root callus. 


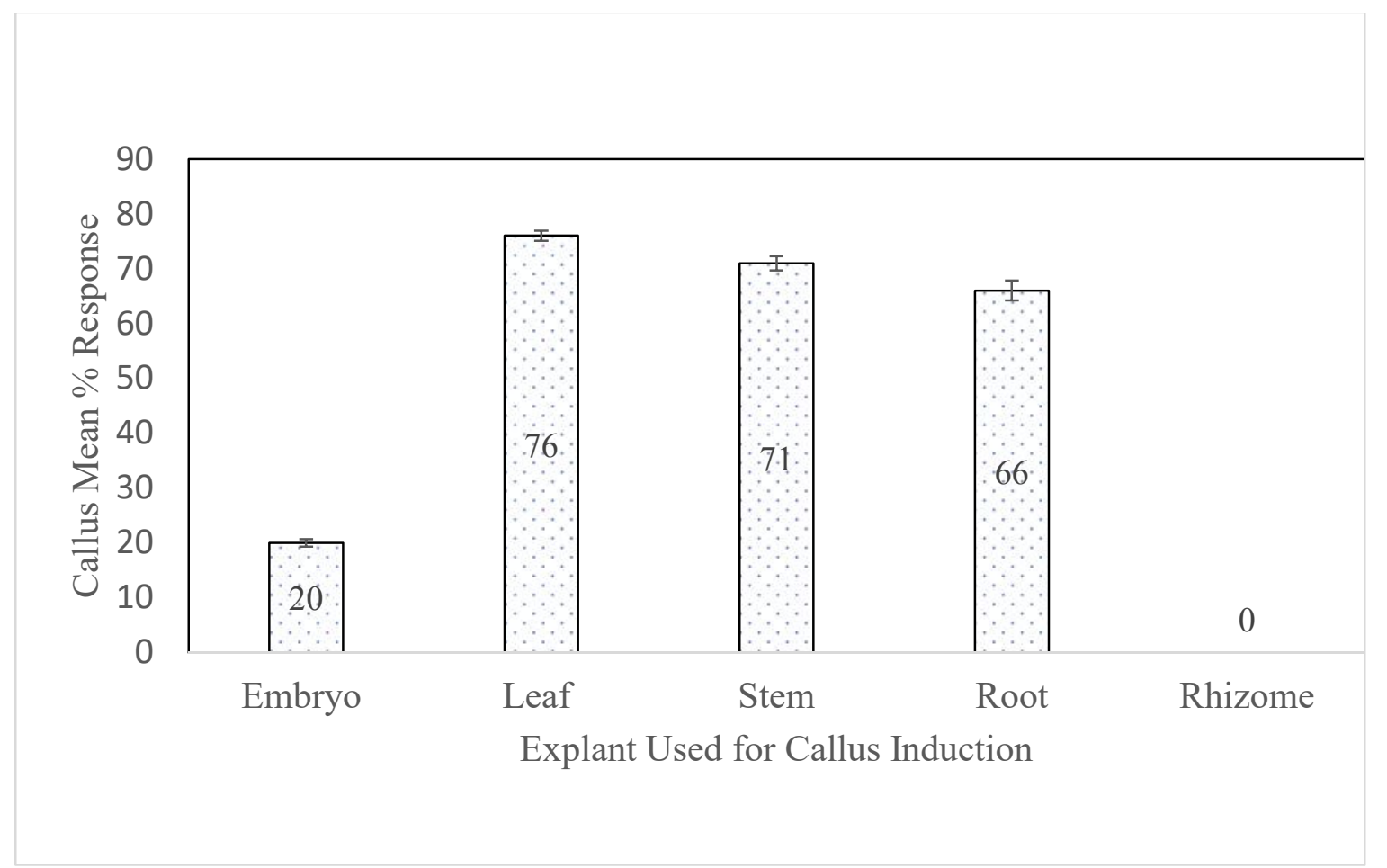

Figure 2. Mean percentage response of callus induction from all five explants used in this study. Embryo: $20 \%$; Leaf: $76 \%$; Stem: $71 \%$; Root: $66 \%$; Rhizome: $0 \%$.

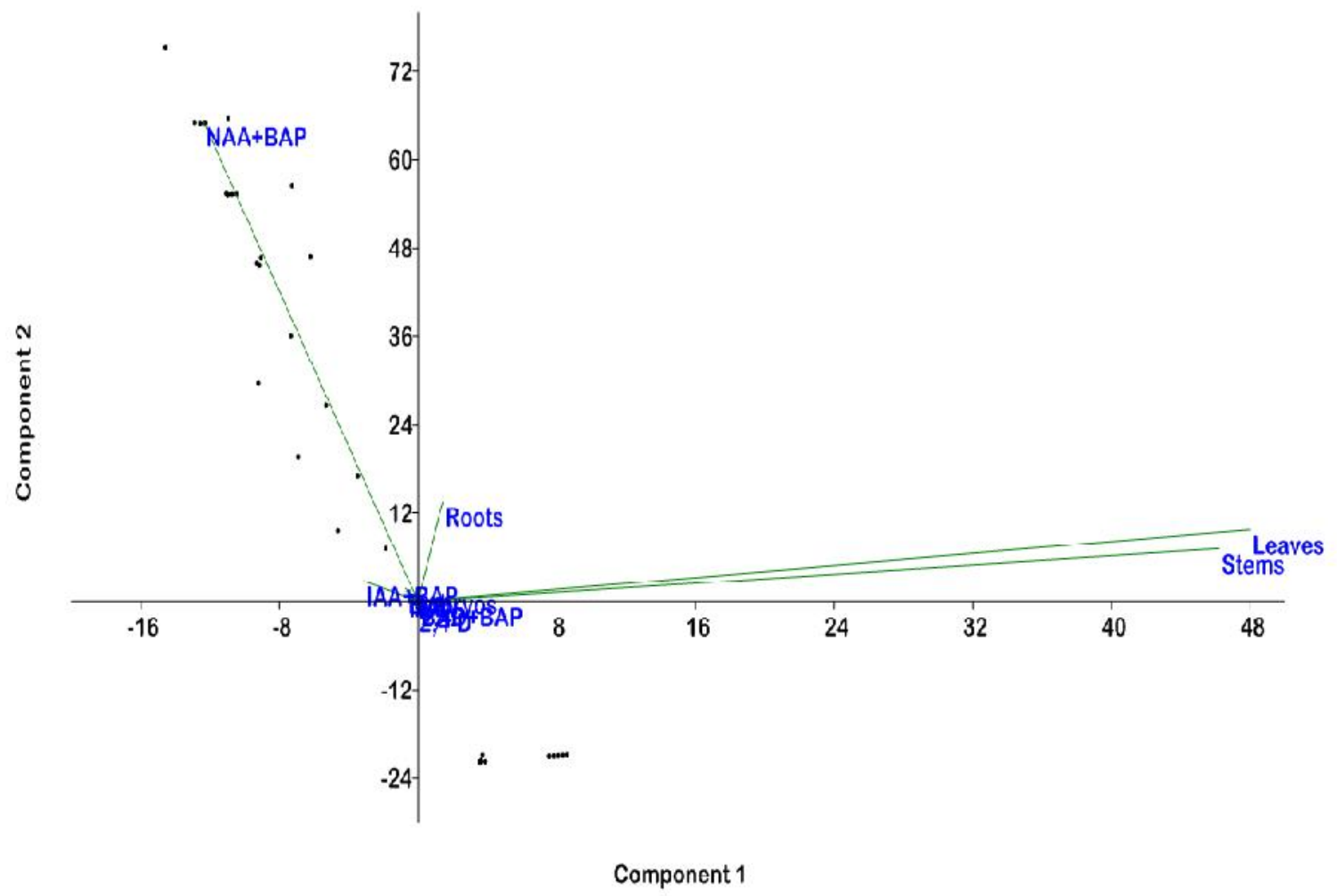

Figure 3. Principal components analysis (PCA) for calli \% induction from all explants (leaf, stem, roots and embryo) and observable response of PGR's (NAA, IAA, BAP, 2,4-D) and their combinations (2,4-D + BAP, NAA + BAP and IAA + BAP). 


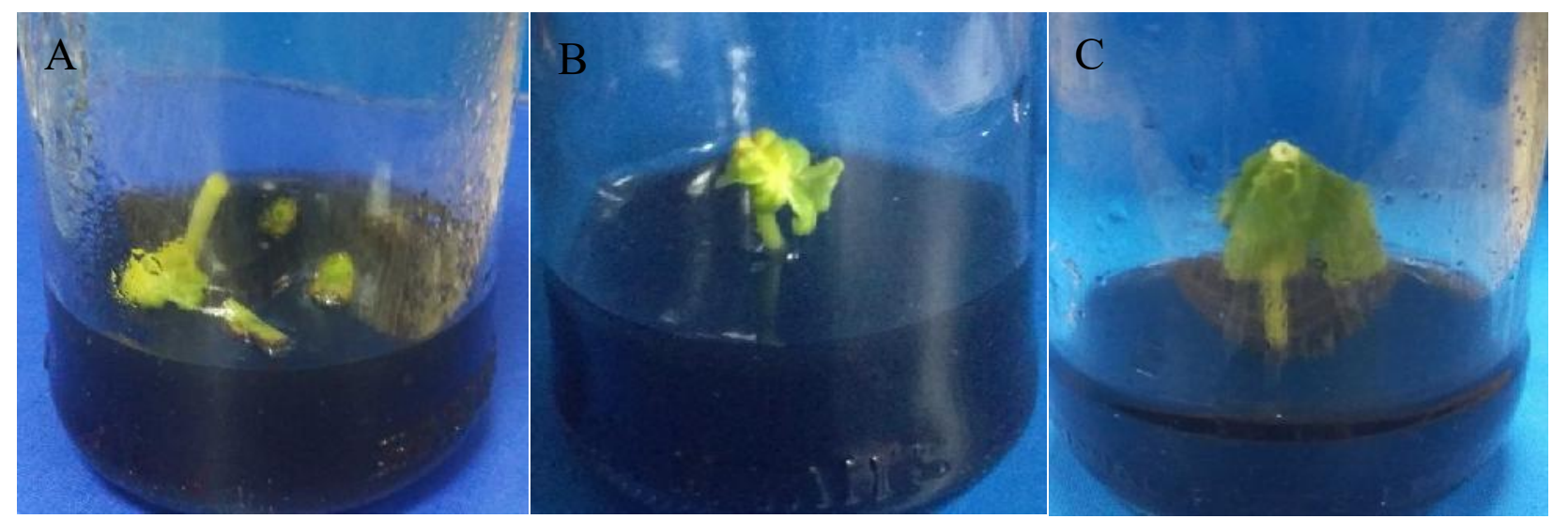

Figure 4. Direct regeneration from rhizome. (A) Initiation of stem from rhizome explant, (B) Initiated shoot transferred to new media, (C) In vitro seedling of $P$. hexandrum.

Table 1. Callus induction from embryos explants on media with different concentrations and combinations of hormones.

\begin{tabular}{cccc}
\hline S/N & Media Code & PGR's & Concentration $(\boldsymbol{\mu M})$ \\
\hline 1 & $\mathrm{MS}$ & - & - \\
2 & $\mathrm{C}_{1 \mathrm{E}}{ }^{*}$ & $\mathrm{BAP}$ & 2.0 \\
3 & $\mathrm{C}_{2 \mathrm{E}}$ & $\mathrm{BAP}$ & 3.0 \\
4 & $\mathrm{C}_{3 \mathrm{E}}$ & $\mathrm{BAP}$ & 4.0 \\
5 & $\mathrm{C}_{4 \mathrm{E}}$ & $2,4-\mathrm{D}$ & 2.0 \\
6 & $\mathrm{C}_{5 \mathrm{E}}$ & $2,4-\mathrm{D}$ & 3.0 \\
7 & $\mathrm{C}_{6 \mathrm{E}}$ & $2,4-\mathrm{D}$ & 4.0 \\
8 & $\mathrm{C}_{7 \mathrm{E}}$ & $2,4-\mathrm{D}+\mathrm{BAP}$ & $2.0+4.0$ \\
9 & $\mathrm{C}_{8 \mathrm{E}}$ & $2,4-\mathrm{D}+\mathrm{BAP}$ & $2.5+4.0$ \\
10 & $\mathrm{C}_{9 \mathrm{E}}$ & $2,4-\mathrm{D}+\mathrm{BAP}$ & $3.0+4.0$ \\
11 & $\mathrm{C}_{10 \mathrm{E}}$ & NAA+BAP & $1.0+5.0$ \\
12 & $\mathrm{C}_{11 \mathrm{E}}$ & NAA+BAP & $1.5+5.0$ \\
13 & $\mathrm{C}_{12 \mathrm{E}}$ & NAA+BAP & $2.0+5.0$ \\
14 & $\mathrm{C}_{13 \mathrm{E}}$ & IAA+BAP & $1.0+5.0$ \\
15 & $\mathrm{C}_{14 \mathrm{E}}$ & IAA+BAP & $1.5+5.0$ \\
16 & $\mathrm{C}_{15 \mathrm{E}}$ & IAA+BAP & $2.0+5.0$ \\
\hline
\end{tabular}

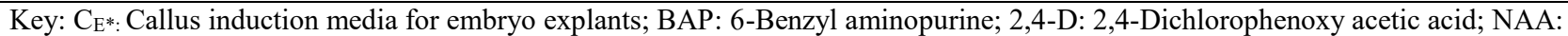
Naphthalene acetic acid; IAA: Indole acetic acid.

Table 2. Callus induction from leaves and stems explants by using different hormonal combinations and concentrations.

\begin{tabular}{cccc}
\hline S/N & Media Code & PGR's & Concentration $(\boldsymbol{\mu M})$ \\
\hline 1 & MS & - & - \\
2 & $\mathrm{C}_{1 L S^{*}}$ & NAA+BAP & $0.75+5.37$ \\
3 & $\mathrm{C}_{2 \mathrm{LS}}$ & NAA+BAP & $1.75+6.37$ \\
4 & $\mathrm{C}_{3 \mathrm{LS}}$ & NAA+BAP & $2.75+7.37$ \\
5 & $\mathrm{C}_{4 \mathrm{LS}}$ & NAA+BAP & $3.75+8.37$ \\
6 & $\mathrm{C}_{5 \mathrm{LS}}$ & NAA+BAP & $4.75+9.37$ \\
7 & $\mathrm{C}_{6 \mathrm{LS}}$ & NAA+BAP & $5.75+10.37$ \\
8 & $\mathrm{C}_{7 \mathrm{LS}}$ & NAA+BAP & $6.75+11.37$ \\
9 & $\mathrm{C}_{8 \mathrm{LS}}$ & NAA+BAP & $7.75+12.37$ \\
10 & $\mathrm{C}_{9 \mathrm{LS}}$ & NAA+BAP & $8.75+13.37$ \\
11 & $\mathrm{C}_{10 \mathrm{LS}}$ & NAA+BAP & $9.75+14.37$ \\
\hline
\end{tabular}

Key: $\mathrm{C}_{\mathrm{LS}}$ : Callus induction media for leaf and stem explants; NAA: Naphthalene acetic acid; BAP: 6-Benzyl aminopurine. 
Table 3. Callus induction from root and rhizome explants by using different hormonal concentrations and combinations.

\begin{tabular}{cccc}
\hline S/N & Media Code & PGR's & Concentration $(\boldsymbol{\mu M})$ \\
\hline 1 & $\mathrm{MS}$ & - & - \\
2 & $\mathrm{C}_{1 \mathrm{R}^{*}}$ & $\mathrm{IAA}$ & 2.5 \\
3 & $\mathrm{C}_{2 \mathrm{R}}$ & $\mathrm{IAA}$ & 5.0 \\
4 & $\mathrm{C}_{3 \mathrm{R}}$ & $\mathrm{NAA}$ & 2.5 \\
5 & $\mathrm{C}_{4 \mathrm{R}}$ & $\mathrm{NAA}$ & 5.0 \\
6 & $\mathrm{C}_{5 \mathrm{R}}$ & $\mathrm{NAA}+\mathrm{BAP}$ & $0.5+5.0$ \\
7 & $\mathrm{C}_{6 \mathrm{R}}$ & $\mathrm{NAA}+\mathrm{BAP}$ & $1.0+5.0$ \\
8 & $\mathrm{C}_{7 \mathrm{R}}$ & $\mathrm{NAA}+\mathrm{BAP}$ & $2.0+5.0$ \\
9 & $\mathrm{C}_{8 \mathrm{R}}$ & $\mathrm{IAA}+\mathrm{BAP}$ & $0.5+5.0$ \\
10 & $\mathrm{C}_{9 \mathrm{R}}$ & IAA+BAP & $1.0+5.0$ \\
11 & $\mathrm{C}_{10 \mathrm{R}}$ & IAA+BAP & $2.0+5.0$ \\
\hline
\end{tabular}

Key: $\mathrm{C}_{\mathrm{R}}$ : Callusing media for rhizome and root explants; - :MS without hormones; IAA: Indole acetic acid; NAA: Naphthalene acetic acid; BAP: 6-Benzyl aminopurine.

Table 4. Shooting media fortified with activated charcoal and different concentrations of auxins (IAA).

\begin{tabular}{ccccc}
\hline S/N & Media Code & PGR's & Concentration $(\boldsymbol{\mu M})$ & Activated Charcoal (g/L) \\
\hline 1 & $\mathrm{MS}$ & - & - & - \\
2 & $* \mathrm{~S}_{1}$ & IAA & 8.42 & 5 \\
3 & $\mathrm{~S}_{2}$ & IAA & 9.42 & 5 \\
4 & $\mathrm{~S}_{3}$ & IAA & 10.42 & 5 \\
5 & $\mathrm{~S}_{4}$ & IAA & 11.42 & 5 \\
6 & $\mathrm{~S}_{5}$ & IAA & 12.42 & 5 \\
\hline
\end{tabular}

Key: *S: Shooting media; IAA: Indole acetic acid.

Table 5. Different hormonal concentrations for rooting media.

\begin{tabular}{ccccc}
\hline S/N & $\begin{array}{c}\text { Media Code } \\
(* \mathrm{R})\end{array}$ & PGR's & Concentration $(\boldsymbol{\mu M})$ & Activated Charcoal $(\mathbf{g} / \mathbf{L})$ \\
\hline 1 & $\mathrm{MS}$ & - & - & - \\
2 & $\mathrm{R}_{1}$ & NAA & 5.0 & 5 \\
3 & $\mathrm{R}_{2}$ & IAA & 5.0 & 5 \\
4 & $\mathrm{R}_{3}$ & BAP+ NAA & $0.5+5$ & 1 \\
5 & $\mathrm{R}_{4}$ & BAP+NAA & $0.5+5.5$ & 1 \\
6 & $\mathrm{R}_{5}$ & BAP+IAA & $0.5+5.0$ & 1 \\
7 & $\mathrm{R}_{6}$ & BAP+IAA & $0.5+5.5$ & 1 \\
\hline
\end{tabular}

Key: *R: Rooting media; :MS without hormones; IAA: Indole acetic acid; NAA: Naphthalene acetic acid; BAP: 6-Benzyl aminopurine.

Table 6. Ex-situ germination of $P$. hexandrum seeds by application of GA3 and conc. $\mathrm{H}_{2} \mathrm{SO}_{4}$.

\begin{tabular}{|c|c|c|c|c|c|}
\hline \multirow[b]{2}{*}{$\mathbf{S} / \mathbf{N}$} & \multirow{2}{*}{$\begin{array}{c}\text { No. of Seeds } \\
\text { under observation }\end{array}$} & \multirow{2}{*}{$\begin{array}{l}\text { Pre-treatments by } \\
\text { PGR's/ chemicals }\end{array}$} & \multicolumn{2}{|c|}{ Seed germination percentage $(\%)$} & \multirow{2}{*}{$\begin{array}{l}\text { Seed germination from } \\
1-8 \text { weeks total \%age }\end{array}$} \\
\hline & & & From 1-4 weeks & From 5-8 weeks & \\
\hline 1 & 30 & - & - & 60 & 60 \\
\hline 2 & 30 & $\mathrm{GA}_{3}$ & 50 & 40 & 90 \\
\hline 3 & 30 & Conc. $\mathrm{H}_{2} \mathrm{SO}_{4}$ & 25 & 50 & 75 \\
\hline
\end{tabular}

Key: GA3: Gibberellic acid; Conc. $\mathrm{H}_{2} \mathrm{SO}_{4}$ : Concentrated Sulfuric acid. 
Table 7. Response of leaf and stem explants to callogenesis on MS media fortified with different concentrations and combinations of PGR's.

\begin{tabular}{|c|c|c|c|c|c|c|c|c|c|c|c|c|c|c|c|}
\hline \multirow{2}{*}{$\begin{array}{l}\text { Media } \\
\text { code }\end{array}$} & \multirow{2}{*}{$\begin{array}{l}\text { Explant } \\
\text { source }\end{array}$} & \multirow{2}{*}{$\begin{array}{c}\text { No. of } \\
\text { explants }\end{array}$} & \multirow{2}{*}{$\begin{array}{c}\text { Days } 1^{\text {st }} \\
\text { callus } \\
\text { observed }\end{array}$} & \multirow{2}{*}{$\begin{array}{c}\text { Calli } \\
\text { induced }\end{array}$} & \multicolumn{8}{|c|}{ Weekly response of callus growth } & \multirow[t]{2}{*}{ Color of calli } & \multirow{2}{*}{$\begin{array}{l}\text { Texture of } \\
\text { calli }\end{array}$} & \multirow{2}{*}{$\begin{array}{l}\text { Percentage } \\
\text { response } \\
(\%)\end{array}$} \\
\hline & & & & & $1^{\text {st }}$ & $2^{\text {nd }}$ & $3^{\text {rd }}$ & $4^{\text {th }}$ & $5^{\text {th }}$ & $6^{\text {th }}$ & $7^{\text {th }}$ & $8^{\text {th }}$ & & & \\
\hline \multirow[t]{2}{*}{ MS } & Leaf & 10 & - & - & - & - & - & - & - & - & - & - & - & - & - \\
\hline & Stem & 10 & - & & - & - & - & - & - & - & - & - & - & - & - \\
\hline \multirow[t]{2}{*}{$\mathrm{C}_{1 \mathrm{LS}}$} & Leaf & 10 & 21 & 4 & - & - & $*$ & ++ & +++ & ++++ & +++++ & +++ & Brownish green & Compact & 40 \\
\hline & Stem & 10 & 28 & 30 & - & - & - & * & ++ & +++ & ++++ & ++++ & $\begin{array}{l}\text { Brown with } \\
\text { creamy texture }\end{array}$ & Fragile & 30 \\
\hline \multirow[t]{2}{*}{$\mathrm{C}_{2 \mathrm{LS}}$} & Leaf & 10 & 19 & 5 & - & - & - & + & +++ & ++++ & +++++ & ++++ & Bright green & Compact & 50 \\
\hline & Stem & 10 & 26 & 6 & - & - & - & + & ++ & +++ & ++++ & ++++ & Yellowish green & Fragile & 60 \\
\hline \multirow[t]{2}{*}{$\mathrm{C}_{3 \mathrm{LS}}$} & Leaf & 10 & 17 & 7 & - & - & - & ++ & ++ & +++ & ++++ & ++++ & Dark green & compact & 70 \\
\hline & Stem & 10 & 26 & 7 & - & - & - & ++ & +++ & ++++ & +++++ & ++++ & Yellowish green & Fragile & 70 \\
\hline \multirow[t]{2}{*}{$\mathrm{C}_{4 \mathrm{LS}}$} & Leaf & 10 & 19 & 8 & - & - & - & ++ & +++ & ++++ & +++++ & ++++ & Light green & Compact & 80 \\
\hline & Stem & 10 & 25 & 7 & - & - & - & + & +++ & ++++ & ++++ & ++++ & $\begin{array}{l}\text { Brown with } \\
\text { creamy texture }\end{array}$ & Compact & 70 \\
\hline \multirow[t]{2}{*}{$\mathrm{C}_{5 \mathrm{LS}}$} & Leaf & 10 & 17 & 8 & - & - & + & ++ & +++ & ++++ & +++++ & ++++ & Green & Compact & 80 \\
\hline & Stem & 10 & 26 & 8 & - & - & - & + & ++ & ++++ & +++++ & ++++ & Pale green & Compact & 80 \\
\hline \multirow[t]{2}{*}{$\mathrm{C}_{6 \mathrm{LS}}$} & Leaf & 10 & 15 & 9 & - & - & $*$ & ++ & +++ & ++++ & +++++ & ++++ & Green & Compact & 90 \\
\hline & Stem & 10 & 28 & 9 & - & - & - & $*$ & ++ & ++++ & +++++ & ++++ & Creamy white & Compact & 90 \\
\hline \multirow[t]{2}{*}{$\mathrm{C}_{7 \mathrm{LS}}$} & Leaf & 10 & 19 & 10 & - & - & ++ & ++ & +++ & ++++ & +++++ & ++++ & Green & Compact & 100 \\
\hline & Stem & 10 & 23 & 9 & - & - & + & ++ & +++ & ++++ & +++++ & ++++ & Creamy white & Compact & 90 \\
\hline \multirow[t]{2}{*}{ C8LS } & Leaf & 10 & 16 & 9 & - & - & + & ++ & +++ & ++++ & +++++ & ++++ & Green & Compact & 90 \\
\hline & Stem & 10 & 21 & 8 & - & - & $*$ & ++ & ++ & +++ & ++++ & +++ & Pale green & Compact & 80 \\
\hline \multirow[t]{2}{*}{$\mathrm{C}_{9 \mathrm{LS}}$} & Leaf & 10 & 21 & 8 & - & - & $*$ & ++ & +++ & ++++ & +++++ & +++ & Green & Compact & 80 \\
\hline & Stem & 10 & 25 & 7 & - & - & - & ++ & +++ & ++++ & +++++ & +++ & $\begin{array}{l}\text { Green with } \\
\text { creamy texture }\end{array}$ & Compact & 70 \\
\hline \multirow[t]{2}{*}{$\mathrm{C}_{10 \mathrm{LS}}$} & Leaf & 10 & 25 & 8 & - & - & - & ++ & +++ & +++ & ++++ & +++ & Pale green & Fragile & 80 \\
\hline & Stem & 10 & 29 & 8 & - & - & - & - & ++ & +++ & ++++ & +++ & $\begin{array}{c}\text { Green with } \\
\text { creamy texture }\end{array}$ & Compact & 80 \\
\hline
\end{tabular}

Key: - : No callus; * : Initiation of callus; + : Very small callus; $++:$ Small callus; $+++:$ Medium sized callus; $++++:$ Large callus; +++++:Very large callus 
Table 8. Callus induction response by rhizome and root explants on media with different concentrations and combinations of plant growth regulators.

\begin{tabular}{|c|c|c|c|c|c|c|c|c|c|c|c|c|c|c|c|}
\hline \multirow{2}{*}{$\begin{array}{l}\text { Media } \\
\text { code }\end{array}$} & \multirow{2}{*}{$\begin{array}{c}\text { Explant } \\
\text { source }\end{array}$} & \multirow{2}{*}{$\begin{array}{c}\text { No. of } \\
\text { explants }\end{array}$} & \multirow{2}{*}{$\begin{array}{c}\text { Days } 1^{\text {st }} \\
\text { callus } \\
\text { observed }\end{array}$} & \multirow{2}{*}{$\begin{array}{c}\text { Calli } \\
\text { induced }\end{array}$} & \multicolumn{8}{|c|}{ Weekly response of callus growth } & \multirow{2}{*}{$\begin{array}{l}\text { Color of } \\
\text { calli }\end{array}$} & \multirow{2}{*}{$\begin{array}{l}\text { Texture } \\
\text { of calli }\end{array}$} & \multirow{2}{*}{$\begin{array}{c}\text { Percentage } \\
\text { response }(\%)\end{array}$} \\
\hline & & & & & $1^{\text {st }}$ & $2^{\text {nd }}$ & $3^{\text {rd }}$ & $4^{\text {th }}$ & $5^{\text {th }}$ & $6^{\text {th }}$ & $7^{\text {th }}$ & $8^{\text {th }}$ & & & \\
\hline \multirow[t]{2}{*}{ MS } & Rhizome & 10 & - & - & - & - & - & - & - & - & - & - & - & - & - \\
\hline & Root & 10 & - & - & - & - & - & - & - & - & - & - & - & - & - \\
\hline \multirow[t]{2}{*}{$\mathrm{C}_{1 \mathrm{R}}$} & Rhizome & 10 & - & - & - & - & - & - & - & - & - & - & - & - & - \\
\hline & Root & 10 & - & - & - & - & - & - & - & - & - & - & - & - & - \\
\hline \multirow[t]{2}{*}{$\mathrm{C}_{2 \mathrm{R}}$} & Rhizome & 10 & - & - & - & - & - & - & - & - & - & - & - & - & - \\
\hline & Root & 10 & - & - & - & - & - & - & - & - & - & - & - & - & - \\
\hline \multirow[t]{2}{*}{$\mathrm{C}_{3 \mathrm{R}}$} & Rhizome & 10 & - & - & - & - & - & - & - & - & - & - & - & - & - \\
\hline & Root & 10 & - & - & - & - & - & - & - & - & - & - & - & - & - \\
\hline \multirow[t]{2}{*}{$\mathrm{C}_{4 \mathrm{R}}$} & Rhizome & 10 & - & - & - & - & - & - & - & - & - & - & - & - & - \\
\hline & Root & 10 & - & - & - & - & - & - & - & - & - & - & - & - & - \\
\hline \multirow[t]{2}{*}{$\mathrm{C}_{5 \mathrm{R}}$} & Rhizome & 10 & - & - & - & - & - & - & - & - & - & - & - & - & - \\
\hline & Root & 10 & 28 & 5 & - & - & - & $*$ & ++ & +++ & ++++ & +++ & $\begin{array}{l}\text { Brown } \\
\text { with } \\
\text { creamy } \\
\text { texture }\end{array}$ & Fragile & 30 \\
\hline \multirow[t]{2}{*}{$\mathrm{C}_{6 \mathrm{R}}$} & Rhizome & 10 & - & - & - & - & - & - & - & - & - & - & - & - & - \\
\hline & Root & 10 & 25 & 6 & - & - & - & - & $*$ & ++ & +++ & +++ & $\begin{array}{c}\text { Brown } \\
\text { with } \\
\text { creamy } \\
\text { texture }\end{array}$ & Fragile & 50 \\
\hline \multirow[t]{2}{*}{$\mathrm{C}_{7 \mathrm{R}}$} & Rhizome & 10 & - & - & - & - & - & - & - & - & - & - & - & - & - \\
\hline & Root & 10 & 25 & 5 & - & - & - & - & + & ++ & +++ & ++++ & $\begin{array}{l}\text { Light } \\
\text { brown }\end{array}$ & Compact & 40 \\
\hline \multirow[t]{2}{*}{$\mathrm{C}_{8 \mathrm{R}}$} & Rhizome & 10 & - & - & - & - & - & - & - & - & - & - & - & - & - \\
\hline & Root & 10 & 27 & 6 & - & - & - & * & ++ & +++ & ++++ & +++ & $\begin{array}{l}\text { Creamy } \\
\text { white }\end{array}$ & Compact & 60 \\
\hline \multirow[t]{2}{*}{$\mathrm{C}_{9 \mathrm{R}}$} & Rhizome & 10 & - & - & - & - & - & - & - & - & - & - & - & - & - \\
\hline & Root & 10 & 30 & 8 & - & - & - & - & $*$ & ++ & ++++ & ++++ & $\begin{array}{l}\text { Brown } \\
\text { with } \\
\text { creamy } \\
\text { texture }\end{array}$ & Compact & 80 \\
\hline \multirow{2}{*}{$\mathrm{C}_{10 \mathrm{R}}$} & Rhizome & 10 & - & - & - & - & - & - & - & - & - & - & - & - & - \\
\hline & Root & 10 & 29 & 7 & - & - & - & - & - & + & ++ & +++ & $\begin{array}{c}\text { Brown } \\
\text { with } \\
\text { creamy } \\
\text { texture }\end{array}$ & Fragile & 70 \\
\hline
\end{tabular}


Table 9. Embryo explants response to callus induction on MS media fortified with different combinations and concentrations of PGR's.

\begin{tabular}{|c|c|c|c|c|c|c|c|c|c|c|c|c|c|c|c|}
\hline \multirow{2}{*}{$\begin{array}{l}\text { Media } \\
\text { code }\end{array}$} & \multirow{2}{*}{$\begin{array}{l}\text { Explant } \\
\text { source }\end{array}$} & \multirow{2}{*}{$\begin{array}{c}\text { No. of } \\
\text { explants }\end{array}$} & \multirow{2}{*}{$\begin{array}{c}\text { Formed } \\
\text { calli }\end{array}$} & \multirow{2}{*}{$\begin{array}{c}\text { Days first } \\
\text { callus } \\
\text { appeared }\end{array}$} & \multicolumn{8}{|c|}{ Weekly response of callus growth } & \multirow[t]{2}{*}{ Color of calli } & \multirow{2}{*}{$\begin{array}{c}\text { Texture of } \\
\text { calli }\end{array}$} & \multirow{2}{*}{$\begin{array}{c}\text { Percentage } \\
\text { response }\end{array}$} \\
\hline & & & & & $1^{\text {st }}$ & $2^{\text {nd }}$ & $3^{\text {rd }}$ & $4^{\text {th }}$ & $5^{\text {th }}$ & $6^{\text {th }}$ & $7^{\text {th }}$ & $8^{\text {th }}$ & & & \\
\hline MS & Embryo & 5 & - & - & - & - & - & - & - & - & - & - & - & - & - \\
\hline $\mathrm{C}_{1 \mathrm{E}}$ & Embryo & 5 & - & - & - & - & - & - & - & - & - & - & - & - & - \\
\hline $\mathrm{C}_{2 \mathrm{E}}$ & Embryo & 5 & - & - & - & - & - & - & - & - & - & - & - & - & - \\
\hline $\mathrm{C}_{3 \mathrm{E}}$ & Embryo & 5 & - & - & - & - & - & - & - & - & - & - & - & - & - \\
\hline $\mathrm{C}_{4 \mathrm{E}}$ & Embryo & 5 & - & - & - & - & - & - & - & - & - & - & - & - & - \\
\hline $\mathrm{C}_{5 \mathrm{E}}$ & Embryo & 5 & 1 & 21 & - & - & $*$ & + & - & - & - & - & Dark brown & Fragile & 20 \\
\hline $\mathrm{C}_{6 \mathrm{E}}$ & Embryo & 5 & 1 & 21 & - & - & $*$ & + & - & - & - & - & Light brown & Fragile & 20 \\
\hline $\mathrm{C}_{7 \mathrm{E}}$ & Embryo & 5 & - & - & - & - & - & - & - & - & - & - & - & - & - \\
\hline $\mathrm{C}_{8 \mathrm{E}}$ & Embryo & 5 & - & - & - & - & - & - & - & - & - & - & - & - & - \\
\hline $\mathrm{C}_{9 \mathrm{E}}$ & Embryo & 5 & - & - & - & - & - & - & - & - & - & - & - & - & - \\
\hline $\mathrm{C}_{10 \mathrm{E}}$ & Embryo & 5 & - & - & - & - & - & - & - & - & - & - & - & - & - \\
\hline $\mathrm{C}_{11 \mathrm{E}}$ & Embryo & 5 & - & - & - & - & - & - & - & - & - & - & - & - & - \\
\hline $\mathrm{C}_{12 \mathrm{E}}$ & Embryo & 5 & - & - & - & - & - & - & - & - & - & - & - & - & - \\
\hline $\mathrm{C}_{13 \mathrm{E}}$ & Embryo & 5 & - & - & - & - & - & - & - & - & - & - & - & - & - \\
\hline $\mathrm{C}_{14 \mathrm{E}}$ & Embryo & 5 & - & - & - & - & - & - & - & - & - & - & - & - & - \\
\hline $\mathrm{C}_{15 \mathrm{E}}$ & Embryo & 5 & - & - & - & - & - & - & - & - & - & - & - & - & - \\
\hline
\end{tabular}

Key: - : No callus; * : Initiation of callus; + : Very small callus 
Table 10. Shoot induction percentage on different shooting media from rhizome explants by direct regeneration process.

\begin{tabular}{cccccc}
\hline $\begin{array}{c}\text { Media } \\
\text { Type }\end{array}$ & $\begin{array}{c}\text { Explant } \\
\text { Source }\end{array}$ & No. of Explants Used & No. of Shoots & $\begin{array}{c}\text { Shoot Length } \\
\text { (cm) }\end{array}$ & $\begin{array}{c}\text { Shooting Percentage } \\
(\%)\end{array}$ \\
\hline MS & Rhizome & 5 & - & - & - \\
${ }^{*} \mathrm{~S}_{1}$ & Rhizome & 5 & 1 & 1 & 20 \\
$\mathrm{~S}_{2}$ & Rhizome & 5 & 2 & 2 & 40 \\
$\mathrm{~S}_{3}$ & Rhizome & 5 & 4 & 4 & 80 \\
$\mathrm{~S}_{4}$ & Rhizome & 5 & 4 & 4 & 80 \\
$\mathrm{~S}_{5}$ & Rhizome & 5 & 3 & 3 & 60 \\
\hline
\end{tabular}

Key: S*: Shooting media.

Table 11. Roots produced on different rooting media.

\begin{tabular}{ccc}
\hline Media Type & Shoots Inoculated & Rooting \\
\hline MS & 1 & - \\
${ }^{*} \mathrm{R}_{1}$ & 1 & - \\
$\mathrm{R}_{2}$ & 1 & Small roots \\
$\mathrm{R}_{3}$ & 1 & - \\
$\mathrm{R}_{4}$ & 1 & - \\
$\mathrm{R}_{5}$ & 1 & Dense Roots \\
$\mathrm{R}_{6}$ & 1 & Dense Roots \\
\hline
\end{tabular}

Key: *R: Rooting media.

Supplementary Tables

Supplementary Table 1: Analysis of variance (ANOVA) for direct shooting on MS media supplemented with 1 g/L activated charcoal and different concentrations of auxins NAA and IAA alone and in combinations. Analysis was done with PAST software. $P$ value higher than 0.05 indicates no significance difference observed in treatments.

\begin{tabular}{llllll}
\hline & SS & df & M S & F & p(same) \\
\hline Between groups: & 5852.08 & 1 & 5852.08 & 10.94 & 0.007918 \\
Within groups: & 5350.83 & 10 & 535.083 & & \\
Total: & 11202.9 & 11 & & & \\
omega`2: & 0.453 & & & & \\
\hline
\end{tabular}

Levene's test for homogeneity of variance, based on means: $\mathrm{p}(\mathrm{same})=0.001792$

Based on medians: $\mathrm{p}($ same $)=0.002188$

SS: Sum of Squares, df: degree of freedom, MS: Mean square between groups, F: F test value,p: significant value, F crit: F critical value.

\section{DISCUSSION}

The seed germination was enhanced in $P$. hexandrum by pre-treatment with $\mathrm{H}_{2} \mathrm{O}_{2}$ and $\mathrm{GA} 3$, which is in accordance with Kumar et al. (2006). Callus induction and suspension culture were successfully reported experiments (Chattopadhyay et al., 2001). In the present findings, the best explant for callus induction was leaf $(76 \%)$ followed by stem $(71 \%)$ and roots $(66 \%)$ as obtained by Neelakandan and Wang (2012) from leaf than stem or rhizome. Viable callus response was observed in leaf explants cultured on media supplemented with NAA and TDZ (Chakraborty et al., 2010; Lata et al., 2010; Tariq et al., 2015).
Callogenesis from leaf explants was reported with NAA and BAP at lower and higher concentrations (Chakraborty et al., 2010). The excised embryos showed only the callus initiation after 8 weeks without generating calli with 2, 4-D. Th role of 2, 4-D was attributed to induce embryogenesis but differentiation requires absence of 2, 4-D (Bapat and Rao, 1988). The continuous presence of 2, 4-D in medium depletes the endogenous auxin level and inhibits development of somatic embryos (CHOI et al., 1999; Fischer-Iglesias et al., 2001). The compact callus induction from root at 1.0-2.0 $\mu \mathrm{M}$ IAA and $5.0 \mu \mathrm{M}$ BAP of present study is similar to Chattopadhyay et al. (2003) where root cuttings induced callus with IAA $(2 \mathrm{mg} / \mathrm{L})$ and activated charcoal $(5 \mathrm{~g} / \mathrm{L})$ 
after three weeks. Callogenesis from roots with IAA 1.5 $\mathrm{mg} / \mathrm{L}$ and NAA $0.5 \mathrm{mg} / \mathrm{L}$ was also reported (Guo et al., 2012).

Rhizome did not induce callogenesis rather it directly developed into multiple shoots with IAA (11.42 $\mu \mathrm{M})$ and activated charcoal $(5 \mathrm{~g} / \mathrm{L})$. Shoots survived after eight weeks of culture and eventually developed into fully-grown plants after sixteen weeks. Chakraborty et al. (2010) reported that IAA and activated charcoal was effective in shoot regeneration directly rather than through callogenesis showing shooting effects of activated charcoal on growth and regeneration (Constantin et al., 1977; FRIDBORG et al., 1978). IAA and BAP were found the best hormones for in vitro regeneration of $P$. hexandrum (Lata et al., 2010).

Rooting was found to be enhanced with IAA (5.0-5.5 $\mu \mathrm{M})$, BAP $(0.5 \mu \mathrm{M})$ and activated charcoal $(1 \mathrm{~g} / \mathrm{L})$ in current study. Similarly, $0.5 \mathrm{mM}$ IAA was also effective to enhance rooting (Nadeem et al., 2000). The present work deals with callogenesis of $P$. hexandrum from different explants for multiple shooting, regeneration ability of rhizomes and the efficient soil transfer of in vitro cultured plants. The micropropagation of $P$. hexandrum for conservation has been reported by different authors (Nadeem et al., 2000; Sagar and Zafar, 2005).

However, the plant is still endangered, so the present study was concerned to develop and improve the protocol for callogenesis and regeneration from different explants. Moreover, this research work described the effect of different PGR's (IAA, NAA, 2,4-D and BAP) for callus induction from different explants of $P$. hexandrum. The best callus induction was observed from leaf explants (100\%), followed by stem $(90 \%)$, roots $(80$ $\%)$ and excised embryo (20\%). The direct shooting of rhizome was significant $(p=0.007198)$. The current tissue culture optimization is potentially responding to callogenesis and organogenesis so it can be considered a step for $P$. hexandrum conservation and propagation.

Authors' contribution: All authors contributed equally to the manuscript and drafted, read, and approved the final manuscript.

Conflict of interest: The authors declare no conflict of interest.

Acknowledgement: Authors like to thank administration of non-timer forest Ayoubia KP Pakistan for providing this medicinal plant from natural habitat.

\section{REFERENCES}

Bapat, V.A., and P.S. Rao (1988). Sandalwood plantlets from 'synthetic seeds'. Plant Cell Rep. 7(6): 434-436.
Chakraborty, A., D. Bhattacharya, S. Ghanta, and S. Chattopadhyay (2010). An efficient protocol for in vitro regeneration of Podophyllum hexandrum, a critically endangered medicinal plant. Indian J. Biotechnol. 9: 217-220.

Chattopadhyay, S., R. Mehra, A. Srivastava, S. Bhojwani, and V. Bisaria (2003). Effect of major nutrients on podophyllotoxin production in Podophyllum hexandrum suspension cultures. Appl. Microbiol. Biot. 60(5): 541-546.

Chattopadhyay, S., A.K. Srivastava, S.S. Bhojwani, and V.S. Bisaria (2001). Development of suspension culture of Podophyllum hexandrum for production of podophyllotoxin. Biotechnol. Lett. 23(24): 2063-2066.

Chaudhari, S.K., Y. Bibi, and M. Arshad (2014). Podophyllum hexandrum: An endangered medicinal plant from Pakistan. Pure Appl. Biol. 3(1): 19.

Chauhan, R.S, B.P. Nautiyal, and M.C. Nautiyal (2013). Trade of threatened Himalayan medicinal and aromatic plants-socioeconomy, management and conservation issues in Garhwal Himalaya, India. Glob. J. Med. Res. Microbiol. Pathol. 13(2). 818.

(https://medicalresearchjournal.org/index.php/G JMR/article/view/322).

Choi, Y.E., J.W. Kim, and E.S. Yoon (1999). High frequency of plant production via somatic embryogenesis from callus or cell suspension cultures in Eleutherococcus senticosus. Ann. Bot. 83(3): 309-314.

Constantin, M.J, R.R. Henke, and M.A. Mansur (1977). Effect of activated charcoal on callus growth and shoot organogenesis in tobacco. In vitro. 13(5): 293-296.

Dar, S.A., A.R. Yousaf, M.U.H. Balkhi, B.A. Ganai, M. Tantry, and F.A. Bhat (2017). Podophyllum hexandrum ameliorates endosulfan-induced genotoxicity and mutagenicity in freshwater cyprinid fish crucian carp. Pharm. Biol. 55(1): 173-183.

Fischer-Iglesias, C., B. Sundberg, G. Neuhaus, and A.M. Jones (2001). Auxin distribution and transport during embryonic pattern formation in wheat. Plant J. 26(2): 115-129.

Fridborg, G., M. Pedersén, L.E. Landström, and T. Eriksson (1978). The effect of activated charcoal on tissue cultures: adsorption of metabolites inhibiting morphogenesis. Physiol. Plantarum. 43(2): 104-106.

Giri, A., and M.L. Narasu (2000). Production of podophyllotoxin from Podophyllum hexandrum: a potential natural product for clinically useful anticancer drugs. Cytotechnology. 34(1-2): 1726. 
Guo, Q., J. Zhou, Z. Wang, and H. Yang (2012). In vitro rooting of Podophyllum hexandrum and transplanting technique. Engineering. 4(10): 142-145.

Heyenga, A.G., J.A. Lucas, and P.M. Dewick (1990). Production of tumour-inhibitory lignans in callus cultures of Podophyllum hexandrum. Plant Cell Rep. 9(7): 382-385.

Kharkwal, A.C., R. Kushwaha, and O. Parkash (2008). An efficient method of propagation of Podophyllum hexandrum: an endangered medicinal plant of the Western Himalayas under ex situ conditions. J. Nat. Med. 62(2): 211-216.

Kumar, S., K.V. Prasad, and M.L. Choudhary (2006). Detection of genetic variability among Chrysanthemum radiomutants using RAPD markers. Curr. Sci: 90(8): 1108-1113.

Kumari, A., D. Singh, and S. Kumar (2017). Biotechnological interventions for harnessing podophyllotoxin from plant and fungal species: current status, challenges, and opportunities for its commercialization. Crit. Rev. Biotechnol. 37(6): 739-753.

Lata, H., S. Chandra, and R. Arora. (2010). Medicinal Plant Biotechnology. In Arora. R. (ed.) Biotechnological characterization of different populations of an endangered medicinal herbPodophyllum hexandrum Royle. Defence Research and Development Organization; Dehli (India). $36 \mathrm{p}$

Li, M., L. Ge, T. Kang, P.Sun, H. Xing, and D. Yang (2018). High-elevation cultivation increases anti-cancer podophyllotoxin accumulation in Podophyllum hexandrum. Ind. Crop. Prod. 121: 338-344.

Nadeem, M., L.M.S. Palni, A.N. Purohit, H. Pandey, and S.K. Nandi (2000). Propagation and conservation of Podophyllum hexandrum Royle: an important medicinal herb. Biol. Conserv. 92(1): 121-129.

Pandey, H., S.K. Nandi, A. Kumar, U.T. Palni, and L.M.S. Palni (2007). Podophyllotoxin content in Podophyllum hexandrum Royle plants of known age of seed origin and grown at a lower altitude. Acta Physiol. Plant. 29(2): 121-126.

Pandey, V., I.D. Bhatt, and S.K. Nandi (2019). Environmental stresses in Himalayan medicinal plants: research needs and future priorities. Biodivers. Conserv. 28(8-9): 2431-2455.

Phondani, P.C., R.K. Maikhuri, L.S. Rawat, N.A. Farooquee, C.P. Kala, S.R. Vishvakarma, K.S. Rao, and K.G. Saxena (2010). Ethnobotanical uses of plants among the Bhotiya tribal communities of Niti Valley in Central Himalaya, India. Ethnobot. Res. Appl. 8: 233-244.

Qazi, P.H., A.B.I.L.A Rashid, and S.A. Shawl (2011). Podophyllum hexandrum: a versatile medicinal plant. Int. J. Pharm. Pharm. Sci. 3: 261-268.

Rajesh, M., G. Sivanandhan, M. Jeyaraj, R. Chackravarthy, M. Manickavasagam, N. Selvaraj, and A. Ganapathi (2014). An efficient in vitro system for somatic embryogenesis and podophyllotoxin production in Podophyllum hexandrum Royle. Protoplasma. 251(5): 12311243.

Sagar, B.P., and R. Zafar (2005). In Vitro.-Enhanced Production of Podophyllotoxin in Phytohormonal-Induced and Regenerated Roots of Podophyllum hexandrum. Pharm. Biol. 43(5): 404-410.

Selvam, A.B.D. (2012). Pharmacognosy of Negative Listed Plants. Botanical Survey of India, Ministry of Environment and Forests; Kolkata (India). 117-127 p

Sharma, J., R. Sharma, and A. Mathur (2013). Protection of Mouse Liver from Gamma Ray Exposure: A Review. Int. J. Pharm. Bio Sci. 4(1): 1011-1026.

Neelakandan, A.K., and K. Wang (2012). Recent progress in the understanding of tissue cultureinduced genome level changes in plants and potential applications. Plant cell rep. 31(4): $597-$ 620.

Sreenivasulu, Y., S.K. Chanda, and P.S. Ahuja (2009). Endosperm delays seed germination in Podophyllum hexandrum Royle-an important medicinal herb. Seed Sci. Technol. 37(1): 10-16.

Sun, P., and M.F. Li (2016). Chilling temperature stimulates growth, gene over-expression and podophyllotoxin biosynthesis in Podophyllum hexandrum royle. Plant Physiol. Biochm. 107: 197-203.

Tariq, A., S. Naz, K. Shahzadi, S. Ilyas, and S. Javed (2015). Study of Genetic Stability in In Vitro conserved Podophyllum hexandrum using RAPD markers. J. Anim. Plant Sci. 25(4): 11141120.

Yang, R., X. Huang, Z. Che, Y. Zhang, and H. Xu (2017). Application of sustainable natural resources in crop protection: Podophyllotoxinbased botanical pesticides derived from Podophyllum hexandrum for controlling cropthreatening insect pests. Ind. Crop. Prod. 107: 45-53. 